\title{
cAMP-responsive element-binding protein expression and regulation in the mouse preimplantation embryo
}

\author{
$X \mathrm{~L} \operatorname{Jin}^{1}$ and $\mathrm{C} \mathrm{O}^{\prime} \mathrm{Neill}^{1,2}$ \\ ${ }^{1}$ Human Reproduction Unit, Discipline of Physiology and ${ }^{2}$ Discipline of Medicine, Royal North Shore Hospital, \\ University of Sydney, Sydney, New South Wales 2065, Australia
}

Correspondence should be addressed to C O'Neill; Email: chriso@med.usyd.edu.au

\begin{abstract}
Gene expression from the new embryonic genome is required for normal preimplantation embryo development. Two members of the cAMP-responsive element-binding protein (Creb) family of transcription factors, Creb1 and activating transcription factor 1 (Atf1), are essential for normal preimplantation development. These transcription factors are activated by phosphorylation. Creb1 mRNA was expressed throughout the preimplantation phase. Cytoplasmic immunolocalization of Creb1 was detected in all preimplantation embryo stages. The antigen was largely excluded from the pronuclei/nuclei at embryonic stages except in the mid-cycle two-cell and compacted eight-cell embryo. Activation-state-specific antibodies showed serine 133 phosphorylated Creb1 localization was similar to Creb1 staining, except that there was no increase in staining at the eight-cell stage. Increased staining of phosphorylated Creb1 was observed in the nucleus of mid-cycle two-cell embryos. Increased expression of phosphorylated Creb1 in the two-cell embryo was induced by brief exposure of embryos to ionomycin, but not by a dibutyryl cAMP. This was blocked by buffering intracellular calcium with 1,2-bis(2-aminophenoxy)ethane- $N, N, N^{\prime}, N^{\prime}$-tetraacetic acid tetrakis (acetoxymethyl ester), but not by a cAMP antagonist, Rp-cyclic $3^{\prime}, 5^{\prime}$ hydrogen phosphorothioate adenosine. Calmodulin is an intracellular receptor for calcium. Calmodulin mRNA was expressed throughout the preimplantation phase of development. The calmodulin antagonist, W-7, inhibited the ionomycin-induced localization of phosphorylated Creb1 in the nucleus. Treatment of embryos with W-7 caused a dose-dependent inhibition of normal development of zygotes to the blastocysts stage. The study shows Creb1 expression and nuclear localization was dynamically regulated in the early embryo. The marked nuclear accumulation and phosphorylation of Creb1 at the two-cell stage occurred at the time of transcription from the embryonic genome and was regulated in a calcium- and calmodulin-dependent manner.

Reproduction (2007) 134 667-675
\end{abstract}

\section{Introduction}

Activation of gene expression from the new embryonic genome is required for normal development and survival of the mammalian preimplantation embryo (Telford et al. 1990, Schultz 1993, Latham \& Schultz 2001). The induction of gene expression requires the action of a range of cis-acting factors, including transcription factors and their cofactors. These factors bind to the regulatory region of genes, resulting in the recruitment of the transcriptional machinery, which includes RNA polymerases. Regulation of transcription factor availability or activity plays an important role in activation and overall regulation of transcription in cells.

The transcription factors that are active within the preimplantation embryo have not been completely defined. Functional genomic analysis shows that two members of the cAMP-responsive element-binding protein (Creb) family of transcription factors are essential for normal preimplantation development (Bleckmann et al. 2002). Deletion of Creb1 and activating transcription factor 1 (Atf1) results in the loss of viability and failure of normal development of peri-implantation mouse embryos (Bleckmann et al. 2002), indicating that these are essential transcription factors for the early embryo.

There is a high degree of functional similarity between the members of the Creb family of transcription factors (Hummler et al. 1994), which comprises Creb1, Atf1, and cAMP-responsive element modulator (Crem). Creb and Atf1 (but not Crem) are expressed in the preimplantation embryo (Bleckmann et al. 2002). These transcription factors possess DNA binding and dimerization motifs (Shaywitz \& Greenberg 1999). They bind to DNA as dimers (Yamamoto et al. 1988), and can form either homodimers with themselves or heterodimers with other members of the family (Kobayashi \& Kawakami 1995). They share the same binding elements within promoters - CRE elements (full-CRE palindrome, TGACGTCA, or half-CRE TGACG/CGTCA). Their capacity for homo- and heterodimerization and the sharing of the same response elements may provide a 
basis for functional compensation or redundancy between Creb1 and Atf1 action in the early embryo and thus account for the early embryonic lethality of Creb1 ${ }^{-/-} A t f 1^{-/-}$compound mutants.

Creb1 was the first transcription factor to be identified as phosphorylation-state dependent, and this applies to all members of the family (for review, see Shaywitz \& Greenberg 1999). In its unphosphorylated state, Creb1 is an ineffective transcription factor (Gonzalez \& Montminy 1989). Phosphorylation was first identified to be induced by cAMP, through the activation of protein kinase A (PKA; Mayr \& Montminy 2001). Inhibition of PKA perturbs both the normal onset of transcription in the two-cell embryo and normal embryo development (Schultz 1989).

Serine 133 is an important phosphorylation site on Creb1 (pCreb1; Mayr \& Montminy 2001). This phosphorylation does not primarily determine the capacity of Creb1 to bind to target DNA elements, but rather promotes recruitment of Creb-binding protein (CBP) and other cofactors to target promoter elements (Chrivia et al. 1993). Phosphorylation results in the exposure of a kinase-inducible domain (KID) in Creb1 (Mayr \& Montminy 2001). This KID is recognized by a Crebbinding domain (KIX) motif in cofactors (Shaywitz \& Greenberg 1999). Upon binding, CBP functions as a 'scaffolding' protein capable of recruiting the transcriptional machinery (Mayr \& Montminy 2001). While PKA is an important kinase for Creb1, it is known that a range of other intracellular kinases, many of which are activated by calcium/calmodulin-dependent pathways, can phosphorylate Creb1 (Mayr \& Montminy 2001). The phosphorylation-state dependence of this transcription factor links its activity to the metabolic state of the cell.

Given the essential requirement for the activation of transcription from the embryonic genome for normal development, the implication of PKA in this process, and the genetic evidence for an essential requirement of the Creb family transcription factors for normal embryo development; this study investigated the expression of Creb1 and the regulation of its serine 133 phosphorylation status during the preimplantation stage of development. The study shows a marked upregulation of phosphorylation of Creb1 in the two-cell embryo and shows that this is primarily regulated by a calcium/calmodulin-dependent pathway.

\section{Results}

Expression of transcription factor Creb1 was assessed by RT-PCR and immunofluorescence. Creb1 mRNA transcripts were detected in the mouse oocyte, zygote, twocell, eight-cell, and blastocyst stages collected from the reproductive tract (Fig. 1A). No transcript was detected when reverse transcriptase or template were omitted from the reaction. Sequence analysis of the product confirmed its identity.
Confocal-fluorescence microscopy detected the presence of Creb1 and pCreb1 in the oocyte and all preimplantation stage embryos collected from the reproductive tract (Fig. $1 \mathrm{~B}$ and $\mathrm{C}$ respectively). Negligible Creb1 staining was observed in nonimmune control samples (two-cell shown as example, Fig. 1Bi). There was uniform cytoplasmic Creb1 staining in oocytes (Fig. 1Bii) and zygotes (Fig. 1Biii); with some exclusion of detectable antigen from the pronuclei when compared with the cytoplasm, there was a relative absence of staining in the nucleoli (Fig. 1Bii and iii). Early two-cell embryos (40 h post-hCG) displayed a predominantly cortical pattern of staining with little staining in the nuclei (Fig. 1Biv). Older two-cell stage embryos (44 h post-hCG) showed a redistribution of staining (Fig. 1Bv), with relatively even staining throughout the cytoplasm but with accumulation of Creb1 staining in the nuclei. Antigen continued to be excluded from the nucleoli. In the uncompacted eight-cell stage embryo (66-68 h post-hCG), the overall level of staining was greater than in two-cell embryos. There was extensive cytoplasmic staining, some of which showed a particulate pattern (Fig. 1Bvi). Nuclear staining was lower than that in the cytoplasm. Compacted eight-cell embryos showed some nuclear accumulation of stain, but this was still generally lower than cytoplasmic staining (Fig. 1Bvii). In blastocysts (88-90 h post-hCG), cytoplasmic staining was less than that observed in eightcell stage embryos and there was a relative exclusion of stain from most nuclei (Fig. 1Bviii).

Nuclear translocation of Creb1 and its transcriptional activation is commonly associated with its serine 133 phosphorylation. We therefore surveyed the same stages of embryo development in embryos collected from the reproductive tract to assess the phosphorylation status of Creb1 during early development. Negligible staining was observed in nonimmune control samples (two cells shown as example, Fig. 1Ci). In the oocyte through the early two-cell stage, there was a pattern of relatively uniform cytoplasmic staining and exclusion of Creb1 from the pronucleus and nucleus (Fig. 1Cii-iv). By $44 \mathrm{~h}$ post-hCG (Fig. 1Cv), the overall level of staining of pCreb1 in two-cell embryos was increased. There was a notable increase in peri-nuclear staining and an accumulation of stain within the nucleus. In the uncompacted and compacted eight-cell embryo, there was uniform staining throughout the embryo (Fig. 1Cvi-vii). In blastocysts, there was a relative exclusion of stain from the nuclei of most cells from the inner cell mass (Fig. 1Cviii).

The nuclear accumulation of Creb1 and pCreb1 staining occurred by $44 \mathrm{~h}$ post-hCG. To assess the factors that control this, $40 \mathrm{~h}$ post-hCG embryos were treated to induce increased cellular activity of cAMP or increased intracellular calcium concentration $\left(\left[\mathrm{Ca}^{2+}\right]_{\mathrm{i}}\right)$ and the nuclear expression of pCreb1 assessed. Two-cell embryos were collected $40 \mathrm{~h}$ post-hCG; they were treated with ionomycin $(1 \mu \mathrm{M})$ for $5 \mathrm{~min}$, db-cAMP 
A Creb1 mRNA

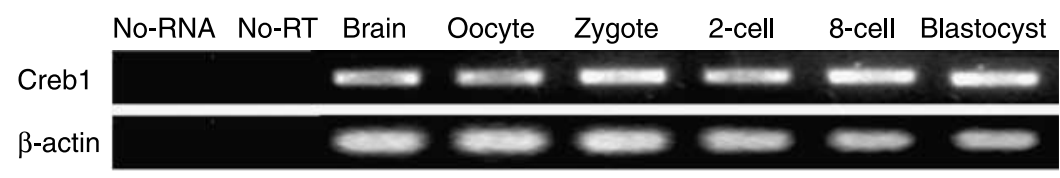

B Creb1
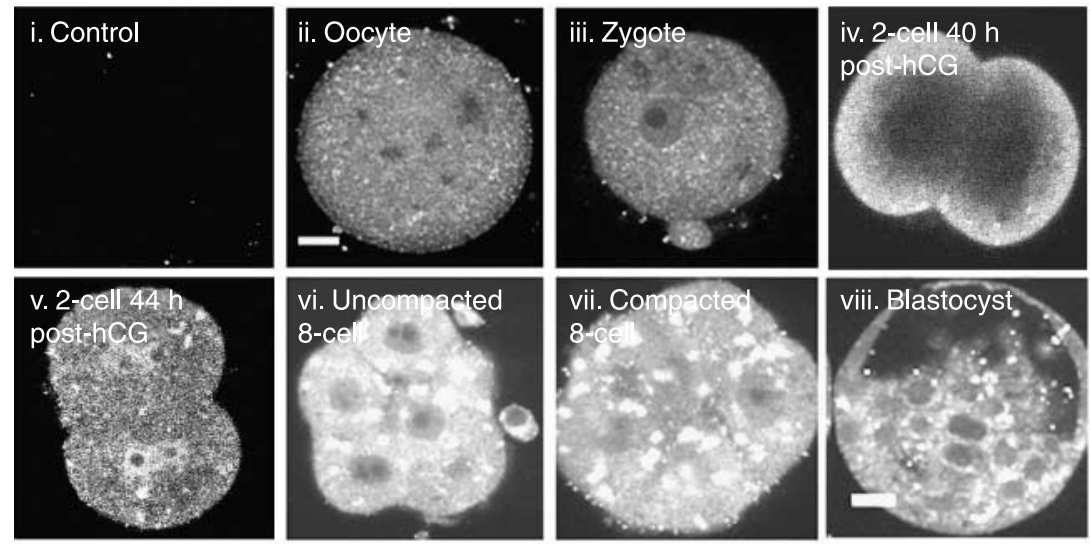

C $\quad$ preb1
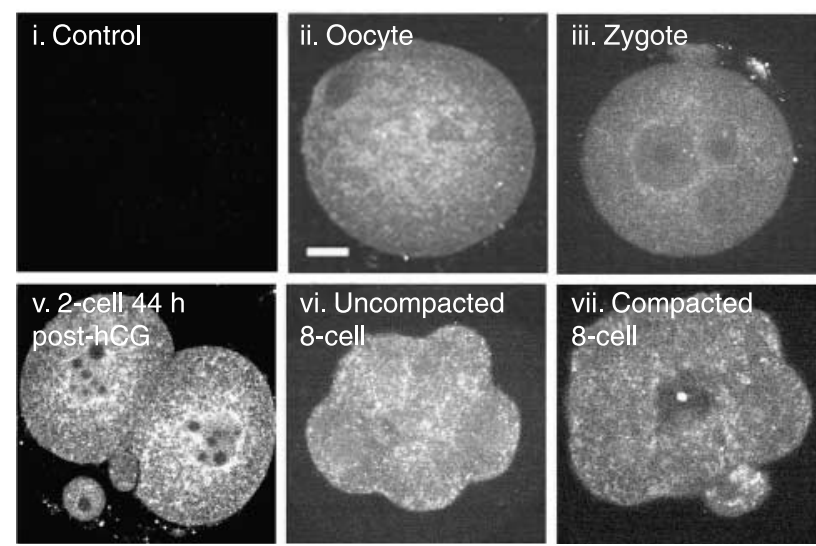

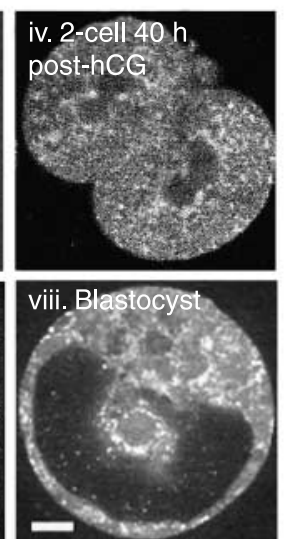

Figure 1 Detection of Creb1 in mouse preimplantation embryos. (A) RT-PCR detected Creb1 transcripts in brain, oocytes, and embryos. Negative controls were the reactions without RNA (No-RNA) and without reverse transcriptase (No-RT). The internal positive control was the expression of $\beta$-actin in embryos. The size of RT-PCR products was 238 bp for $\beta$-actin and 229 bp for Creb1. Each band represented mRNA from two oocytes or embryos. The results are representative of at least three replicates. (B) and (C) Confocal-fluorescent microscopy detection of Creb1 or pCreb1 expression respectively, in mouse preimplantation embryos. Negative control was the staining of nonimmune $\lg G$ instead of primary antibodies, two-cell embryos controls are shown as representative examples. The scale bar represents $10 \mu \mathrm{m}$.
$(50 \mathrm{mM})$ for $30 \mathrm{~min}$, or exposed to vehicle control media. They were then removed and placed in culture media for $5 \mathrm{~min}$, after which they were fixed and stained for pCreb1 and assessed by whole section immunolocalization.

Brief exposure of embryos to ionomycin induced increased pCreb1 staining throughout the two-cell embryo and increased peri-nuclear and nuclear staining (Fig. 2Ai-iii). Treatment with db-cAMP had no obvious effect (Fig. 2Aiv-vi) when compared with vehicle controltreated embryos (Fig. 2Avii-ix). This increase in nuclear staining after ionomycin was statistically significant $(P<0.05$; Fig. 2B). The treatment was repeated and the analysis was performed by confocal optical section microscopy (Fig. 3). This confirmed the overall increase in intensity of pCreb1 staining in response to ionomycin and showed a dominant pattern of increased peri-nuclear and nuclear staining (but the antigen was largely excluded from the nucleoli). The results show representative examples of staining from two individual replicates of the experiment (Fig. 3A). Figure 3B shows a pseudocolor representation of the range of staining intensities observed. Even the smallest increase in pCreb1 staining in response to ionomycin was more intense than that seen in the most intensely stained vehicle control. Quantitative analysis of nuclear staining showed that the ionomycin in treated embryos had approximately fourfold greater staining than controls $(P<0.001$; Fig. 3C). This pattern of staining is similar to the spontaneous change in staining observed at $44 \mathrm{~h}$ post-hCG in two-cell embryos (Fig. 1Bv).

To determine whether the effect of ionomycin was due to the generation of a calcium transient, embryos were 


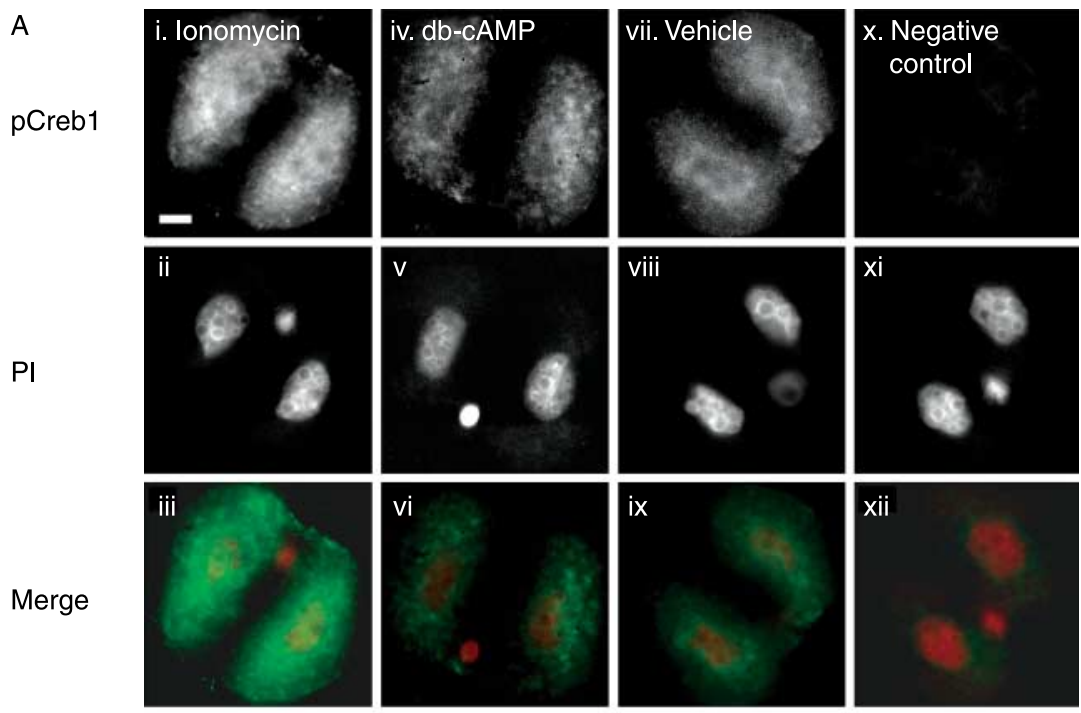

B Nuclear intensity

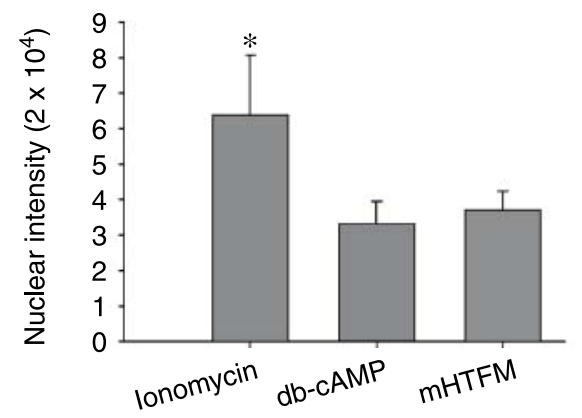

treated with intracellular calcium chelator, BAPTA-AM, to buffer changes in the $\left[\mathrm{Ca}^{2+}\right]_{i}$. Ionomycin was again seen to cause the upregulation and peri-nuclear/nuclear staining, pCreb1 staining when compared with controls (Fig. 4i-ii). This increased nuclear pCreb1 staining was blocked by BAPTA-AM treatment (Fig. 4iii-iv), but was unaffected by the cAMP antagonist, Rp-cAMP (Fig. $4 \mathrm{v}-\mathrm{vi}$ ). This result showed the action of ionomycin was via its capacity to generate $\mathrm{a}\left[\mathrm{Ca}^{2+}\right]_{i}$ transient and is further evidence that CAMP was not required for this increased nuclear localization of pCreb1 in this model.

Calmodulin is an intracellular receptor for calcium, and calmodulin/calmodulin-dependent kinases are important mediators of Creb1 phosphorylation. Calmodulin mRNA was expressed in the oocyte, zygote, two-cell, eight-cell, and blastocyst stages of development (Fig. 5A). The calmodulin antagonist (W-7) blocked ionomycin-induced pCreb1 localization (Fig. 5Bi-iii) in the nucleus. Treatment with $\mathrm{W}-7$ in the absence of ionomycin had no obvious effect on the embryo (Fig. 5Biv). Treatment of embryos with $\mathrm{W}-7$ caused a dose-dependent $(P<0.001)$ inhibition of normal development of zygotes to the blastocysts stage. The inactive form $(\mathrm{W}-5)$ was without effect $(P>0.05$;
Figure 2 The effects of $\mathrm{Ca}^{++}$or cAMP on pCreb1 expression in two-cell embryo. (A) Embryos were treated with ionomycin, db-cAMP, or vehicle as described. They were then stained for pCreb1 and co-stained with propidium iodide (PI). FITC and PI images were merged using Image-Pro Plus. Images are representative. The scale bar represents $10 \mu \mathrm{m}$. (B) Quantitative analysis of staining in 14 embryos in each treatment. ${ }^{*} P<0.05$.

Fig. 5C). The results show that in the two-cell mouse embryo Creb1 phosphorylation and nuclear localization was induced by calcium and calmodulin signals but not by cAMP.

\section{Discussion}

This study shows that Creb1 is expressed in the oocyte and throughout the preimplantation phase of development. Creb1 is a transcription factor and for it to be functional it needs to be localized within the nucleus. Nuclear localization of Creb1 was observed to increase for the first time at the mid-cycle two-cell stage and again at the eight-cell stage. Nuclear localization of phosphorylated Creb1 was also most evident at these stages of development.

This first nuclear localization of Creb1 occurs around the time when definitive transcription from the embryonic genome is thought to be initiated (Latham \& Schultz 2001). The promoters of a large number of genes involved in cell proliferation, survival, and differentiation contain the CRE-element (Creb1-binding site). The nuclear localization of Creb1 at this time may indicate that it is one of the transcription factors involved 
A

Rep 1
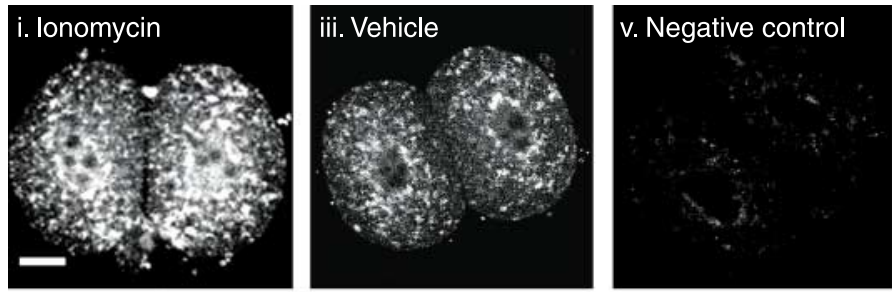

$\operatorname{Rep} 2$
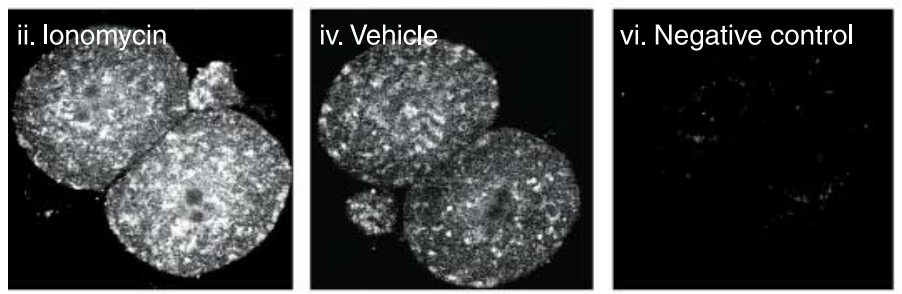

B Pseudo-colourisation

onomycin
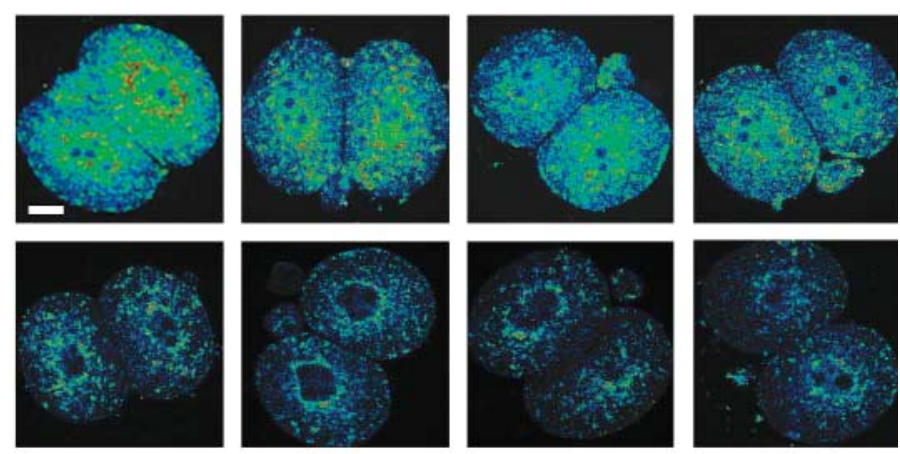

C Nuclear intensity

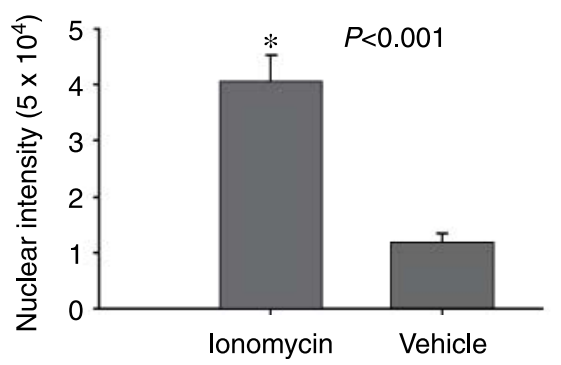

in this first activation of transcription from the embryonic genome. It should be noted, however, that nuclear localization and phosphorylation of Creb1 does not itself show that the transcription factor is active and further functional studies are required to provide evidence of this. The embryopathy that occurs following the genetic deletion of Creb1 and Atf1 (Bleckmann et al. 2002) is consistent with a requirement for Creb1-mediated transcription at these early stages of development.

Serine 133 in Creb1 corresponds to serine 63 of Atf1 and serine 117 of Crem (Shaywitz \& Greenberg 1999). The antibody used in this study to detect pCreb1 also cross-reacts with pAtf1. To date, it has not been possible to produce an antibody that selectively recognizes pCreb1. Thus, we cannot exclude at this time the
Figure 3 Confocal optical sections of pCreb1 expression after treatment with ionomycin. (A) Two-cell embryos were treated with ionomycin or vehicle, and then fixed and stained for pCreb1. Negative controls were stained with nonimmune immunoglobulin. Equatorial optical sections of two representative examples are shown (Rep 1 and 2). The scale bar represents $10 \mu \mathrm{m}$. (B) Pseudocolor representation of the pCreb1 staining. The sections shown represent the range of staining intensities observed in each treatment. (C) Quantitative analysis of staining in seven to ten embryos in each treatment. $* P<0.001$. possibility that the increased staining observed includes some component of pAtf1 expression. It was found that the nuclear localization of staining could be induced by short treatment with the calcium ionophore, ionomycin, and was blocked by buffering intracellular $\left[\mathrm{Ca}^{2+}\right]_{i}$. This analysis shows that the most marked change in Creb1 and pCreb1 expression occurred at the mid-cycle twocell stage, there was a shift from complete exclusion of antigen from the nucleus to a marked nuclear accumulation of this activated transcription factor.

Buffering the calcium transient induced by ionomycin by treatment with BAPTA-AM prevented the accumulation of pCreb1 staining, showing that it was the change in $\left[\mathrm{Ca}^{2+}\right]_{i}$ that induced this change. The cAMP analogue, db-cAMP, had no effect on pCreb1 

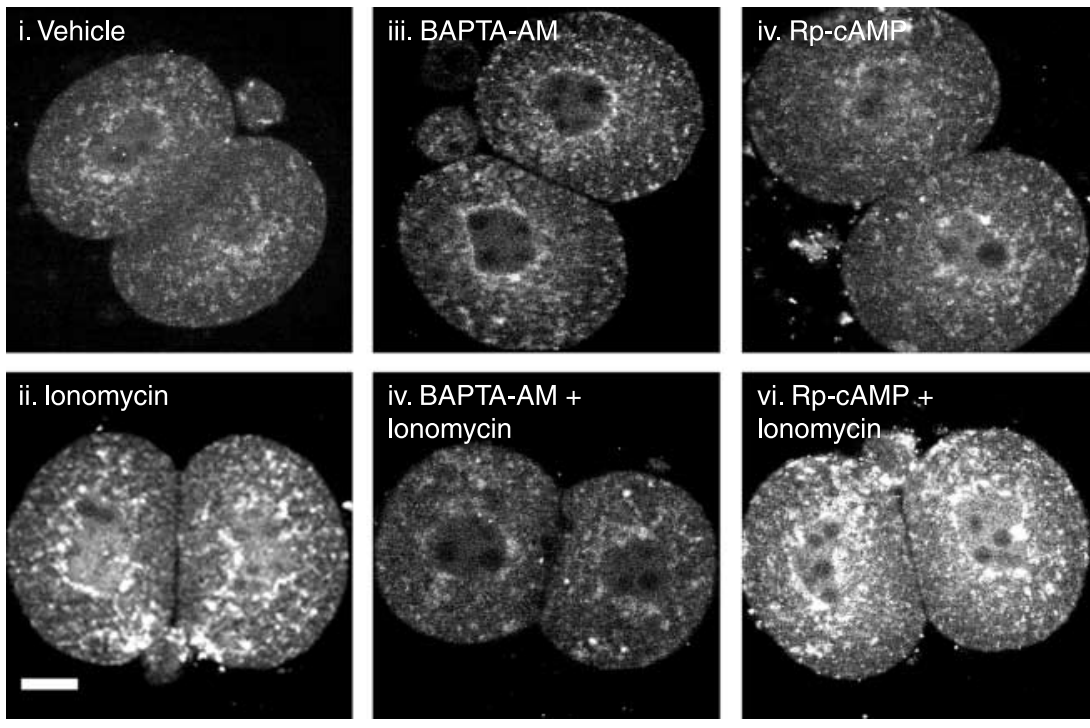

Figure 4 The effect of inhibitors of calcium and cAMP on ionomycin-induced pCreb1 expression. Two-cell embryos were treated with ionomycin or control media in the presence or absence of a calcium chelator (BAPTA-AM) or inhibitor of cAMP action (Rp-cAMP). Equatorial optical confocal sections of representative examples of each treatment are shown. The scale bar represents $10 \mu \mathrm{m}$. nuclear localization in response to ionomycin. An inhibitor of cAMP had no effect on the pCreb1 nuclear localization induced by an ionomycin-induced calcium transient. These results place $\left[\mathrm{Ca}^{2+}\right]_{i}$ transients as an important mechanism in the induction of Creb1 phosphorylation in this model. Calmodulin is an important intracellular receptor of calcium, coupling increased $\left[\mathrm{Ca}^{2+}\right]_{i}$ with a range of downstream effector systems. Inhibition of calmodulin blocked the nuclear pCreb1 staining, and treatment with this antagonist also reduced the capacity of embryos to develop normally, consistent with observations of the role of calmodulin in embryo development and the onset of transcription from the embryonic genome.

\section{A CaM mRNA}

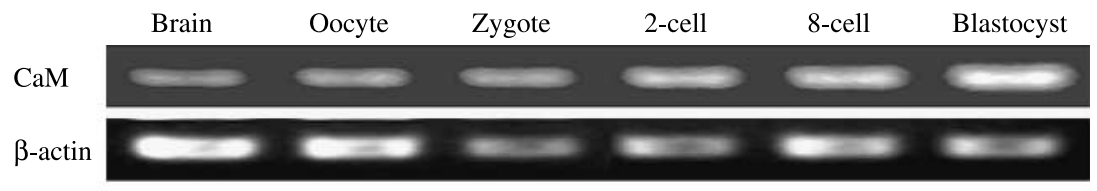

B W-7 on ionomycin-induced pCREB1
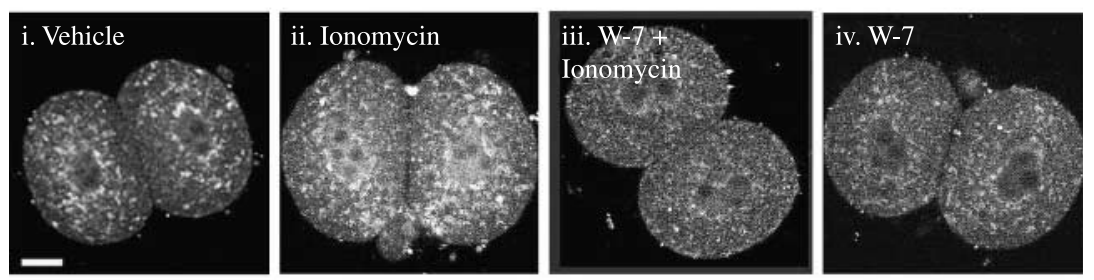

C W-7 on zygote development

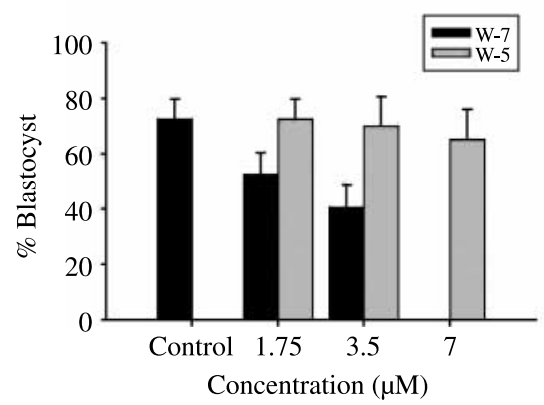

Reproduction (2007) 134 667-675
Figure 5 The expression of calmodulin and its influence on pCreb1 expression in two-cell embryos. (A) RT-PCR detected calmodulin transcripts in brain, oocytes and embryos. Negative controls were the reactions without RNA and without reverse transcriptase (not shown). The internal positive control was the expression of $\beta$-actin. The size of RT-PCR products was $238 \mathrm{bp}$ for $\beta$-actin and 248 bp for calmodulin. Each band represented mRNA from two oocytes or embryos. The results are representative of at least three replicates. (B) The effect of a calmodulin inhibitor on ionomycin-induced pCreb1 expression. Twocell embryos were treated with ionomycin or control media in the presence or absence of a calmodulin inhibitor (W-7). Equatorial optical confocal sections of representative examples of each treatment are shown. The scale bar represents $10 \mu \mathrm{m}$. (C) The effect of incubating zygotes in various concentrations of $\mathrm{W}-7$ or its inactive analogue (W-5) on the proportion of embryos that developed to morphologically normal blastocyst after $96 \mathrm{~h}$. 
Although phosphorylation of Creb1 is not required for nuclear localization, it is required for its effective action as a transcription factor. Upon cellular stimulation, the peak rate of phosphorylation occurs after $30 \mathrm{~min}$ and results in near stoichiometric phosphorylation of Creb1 (Mayr \& Montminy 2001). Phosphorylation at serine 133 results in solvent exposure of the KID region allowing it to interact with the KIX domain within its co-activators, such as CBP and p300. This interaction allows recruitment of cofactors to the promoter (for reviews see Shaywitz \& Greenberg 1999, Mayr \& Montminy 2001). The recruitment of transcriptional co-activators mediates Creb-stimulated activation of target genes through their association with RNA polymerase II complexes (Kee et al. 1996) and intrinsic histone acetyltransferase activity (Korzus et al. 1998).

PKA is a canonical activator of Creb1, and acts by inducing serine 133 phosphorylation (Shaywitz \& Greenberg 1999, Mayr \& Montminy 2001). It is now recognized that a range of other kinases can also exert this action. For instance, calcium/calmodulin-dependent kinases also phosphorylate Creb1 at serine 133 (Mayr \& Montminy 2001). Furthermore, PKA can induce the nuclear translocation of extracellular signal regulated kinases which can then induce Creb1 phosphorylation (Impey et al. 1998). Further investigation is required to determine the transduction pathways that result in the $\left[\mathrm{Ca}^{2+}\right]_{i}$-induced Creb1 phosphorylation observed in this study.

The activation of Creb1 provides a link between signal transduction events and gene expression. This study shows that calcium transients are capable of inducing phosphorylation of Creb1. It is recognized that several events during early embryo develop induce defined calcium signaling events. For example: (1) calcium transients occur in the mouse zygote as a consequence of fertilization and these enhance the viability of embryos (Leach et al. 1993, Stachecki et al. 1994); (2) embryo-derived paf (1-o-alkyl-2-acetyl-snglycero-3-phosphocholine) caused periodic $\left[\mathrm{Ca}^{2+}\right]_{\mathrm{i}}$ transients in the late zygote and mid-cycle two-cell stage, these transients are also necessary for normal survival of embryos in vitro (Emerson et al. 2000, Lu et al. 2004); (3) calcitonin-induced $\left[\mathrm{Ca}^{2+}\right]_{i}$ transients in four-cell to blastocyst stage embryos; and (4) lysophosphatidic acid-induced $\left[\mathrm{Ca}^{2+}\right]_{\mathrm{i}}$ transients in blastocysts, which resulted in transient accumulation of heparinbinding EGF-like growth factor on the blastocyst surface (Liu \& Armant 2004). This study offers the possibility that activation of Creb1 may be a mechanism of transducing these signaling events into altered patterns of Creb1-mediated transcription. In the human, 4084 genes are identified as putative Creb-target genes (Zhang et al. 2005), thus activation of Creb1 has the potential to induce pleiotypic changes in the embryo's expressed transcriptome.
Future studies are required to provide evidence of the transcriptional activity of the nuclear Creb1, identify its target genes, and in particular identify whether it acts as part of the mechanism of initiation of transcription from the zygotic genome. Other important matters for consideration are to characterize the interactions between Creb1 and Atf1 and to fully define the mechanisms that regulate the functional expression of these transcription factors.

\section{Materials and Methods}

\section{Animals}

The use of animals was in accordance with the Australian Code of Practice for the Care and Use of Animals for Scientific Purpose and was approved by the Institutional Animal Care and Ethics Committee. F1 mice (C57BL/6× CBA/He) were used in all experiments. Animals were housed and bred in the Gore Hill Research Laboratory, St Leonards, NSW, Australia. All mice were kept under $12 \mathrm{~h}$ light: $12 \mathrm{~h}$ darkness cycle and had access to food and water ad libitum. Six-week-old females were superovulated by i.p. injection of $5 \mathrm{IU}$ equine chorionic gonadotropin (Folligon; Intervet International, Boxmeer, The Netherlands) followed $48 \mathrm{~h}$ later by $5 \mathrm{IU}$ human chorionic gonadotropin (hCG, Chorulon; Intervet). Females were paired with males of proven fertility. Pregnancy was confirmed by the presence of a copulation plug the following morning (day 1 ).

\section{Mouse embryo collection and culture}

Cumulus masses or embryos were flushed from the reproductive tract with HEPES-buffered modified human tubal fluid medium and cultured in modified human tubal fluid medium (modHTF; O'Neill 1997). All components of the media were tissue culture grade (Sigma Chemical Company) and contained $3 \mathrm{mg} \mathrm{BSA} / \mathrm{ml}$ unless otherwise stated (CSL Ltd, Melbourne, VIC, Australia). Except where otherwise explicitly stated, the results are for embryos collected directly from the reproductive tract were processed without additional culture. Oocytes and zygotes were collected 20-21 h after hCG and freed from their cumulus cells by brief exposure to $300 \mathrm{IU}$ hyaluronidase (Sigma) in HEPES-buffered modHTF. Other stage embryos were collected at $40 \mathrm{~h}$ (two-cell), $44 \mathrm{~h}$ (late two-cell), 66-68 h (eight cell), and 88-90 h (blastocyst) after hCG. Where embryos were subjected to culture, they were recovered in minimal volume and assigned to various treatments as required in modHTF. Embryos were cultured individually in $10 \mu \mathrm{l}$ volumes in 60-well HLA plates (LUX 5260, Nunc, Naperville, IL, USA) overlaid by an $\sim 2 \mathrm{~mm}$ depth of heavy paraffin oil (Sigma). Culture was at $37^{\circ} \mathrm{C}$ in $5 \% \mathrm{CO}_{2}$ for the periods indicated in individual experiments. The developmental stage and morphology of embryos were assessed by visualizing the embryos with an inverted phase contrasted microscope (Nikon Diaphot, Nikon, Japan) at 24-h intervals after zygote collection. 


\section{Pharmacological agents and treatments}

Ionomycin (Calbiochem, MERCK Pty.) and 1,2-bis(2aminophenoxy)ethane- $N, N, N^{\prime}, N^{\prime}$-tetraacetic acid tetrakis (acetoxymethyl ester) (BAPTA-AM; Sigma) were prepared as 2000-fold concentrated stock in dimethyl sulfoxide. They were diluted to working concentrations of $1 \mu \mathrm{M}$ ionomycin and $50 \mu \mathrm{M}$ BAPTA-AM with modHTF. Other agents were prepared at working concentrations by dissolving directly in modHTF on day of use: Rp-cyclic 3',5'-hydrogen phosphorothioate adenosine triethylammonium salt (Rp-cAMP; Sigma); dibutyryl cAMP (db-cAMP, $\mathrm{N}^{6}, 2^{\prime}$-O-dibutyryladenosine $3^{\prime}, 5^{\prime}$-cyclic monophosphate sodium salt; Sigma); and $\mathrm{W}-7$ ( $\mathrm{N}$-(6-aminohexyl)5-chloro-1-naphthalenesulfonamide, $\mathrm{HCl}$; Calbiochem).

To assess the regulation of pCreb1 expression, two-cell embryos were collected $40 \mathrm{~h}$ post-hCG. Embryos were treated with (i) control media alone; (ii) $1 \mu \mathrm{M}$ ionomycin for $5 \mathrm{~min}$; or (iii) $50 \mathrm{mM} \mathrm{db-cAMP}$ for $30 \mathrm{~min}$. Inhibitor treatments were:

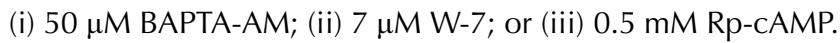
Inhibitors were applied either alone or in conjunction with the ionomycin treatment. After exposure to treatments, embryos were washed three times in modHTF and cultured for a further $5 \mathrm{~min}$ in modHTF alone. Embryos were then fixed and stained.

\section{Reverse transcriptase PCR}

Reverse transcriptase-PCR (RT-PCR) was performed as previously described (Stojanov \& O'Neill 2001). Embryos were collected fresh from the reproductive tract. The embryos were washed three times in cold $\mathrm{Ca}^{2+}-\mathrm{Mg}^{2+}$-free Dubbecco's PBS (Sigma) and then transferred in a minimal volume to $30 \mu \mathrm{PCR}$ buffer $(50 \mathrm{mM} \mathrm{KCl}, 15 \mathrm{mM}$ Tris- $\mathrm{HCl}(\mathrm{pH} 8.0))$ in diethyl pyrocarbonate-treated (Sigma) MilliQ water containing $1 \mathrm{IU}$ RNAse inhibitor (Applied Biosystems, Foster City, CA, USA). The embryos were lyzed by three cycles of freezing in liquid nitrogen and thawing (with vortexing) and subject to RT-PCR. $\mathrm{RT}$ was in $12.5 \mathrm{U}$ murine leukemia virus reverse transcriptase (MuLV), $1 \cup$ RNase inhibitor, $4 \mathrm{mM} \mathrm{MgCl} 2,50 \mathrm{mM} \mathrm{KCl}$, $15 \mathrm{mM}$ Tris- $\mathrm{HCl}$ (pH 8.0), 0.5 mM dNTPs (Applied Biosystems), and $1.5 \mu \mathrm{M}$ allele-specific reverse primer. The reactions were incubated for $10 \mathrm{~min}$ at room temperature, $30 \mathrm{~min}$ at $42{ }^{\circ} \mathrm{C}$, and $2 \mathrm{~min}$ at $99^{\circ} \mathrm{C}$. Two negative controls were included: no RT enzyme and no template to test for extraneous DNA or RNA contaminations respectively. PCR-specific products included 2-4 $\mu \mathrm{l}$ cDNA template (equivalent to 1-2 embryo), $1.5 \mu \mathrm{l}$ Amplitaq Gold DNA polymerase (Applied Biosystems), 2-2.5 mM MgCl $2,50 \mathrm{mM} \mathrm{KCl}, 15 \mathrm{mM}$ Tris- $\mathrm{HCl}$ (pH 8.0), 0.5 mM dNTP (Applied Biosystems), and 5\% (v/v) dimethyl sulfoxide (Sigma), $1.5 \mu \mathrm{M}$ each of gene-specific primers. The reactions were incubated for $10 \mathrm{~min}$ at $94{ }^{\circ} \mathrm{C}$ and 40 cycles of $15 \mathrm{~s}$ at $94{ }^{\circ} \mathrm{C}$ and $1 \mathrm{~min}$ at $58^{\circ} \mathrm{C}$ in a Corbett Thermal Reactor. PCR products were analyzed by electrophoresis on $2 \%(\mathrm{w} / \mathrm{v})$ agarose gel stained with ethidium bromide to visualize PCR products on u.v. transluminator. Fragments were verified by size and representative samples had sequence analyzed (SUPAMAC, Redfern, NSW, Australia). Primers were obtained from Sigma-Genosys (Sigma). $\beta$-Actin was used as a positive control. The sequence of oligonucleotide primers and the product size were as follows: $\beta$-actin
5'-CGTGGGCCGCCCTAGGCACCA, 3'-TTGGCCTTAGGGTTCAGGGGG, 243 bp; Creb1-5'-CCAACCCCCATTTACCAAC-3', 3'-CTGAGGCAGCTTGAACAAC-5', 229 bp; and Calmodulin 5'-AAAGACACAGATAGCGAAGAAG, 3'-GAGGGGGAAAAGTAGTTGAAAG, 248 bp.

\section{Immunofluorescence}

Embryos were washed three times in PBS with $0.1 \%$ BSA, $0.1 \%$ Tween-20, and $0.2 \%(\mathrm{w} / \mathrm{v})$ sodium azide (washing buffer), fixed with $2 \%$ paraformaldehyde (w/v) (Sigma) for $30 \mathrm{~min}$ and then permeabilized with $2 \%$ paraformaldehyde containing $0.3 \%$ Tween-20 (Sigma) at room temperature for $30 \mathrm{~min}$. Embryos were washed three times in washing buffer, blocked in $2 \% \mathrm{BSA}$, and $30 \%$ serum for $3 \mathrm{~h}$, and stained overnight at $4{ }^{\circ} \mathrm{C}$ with primary antibodies: $4 \mu \mathrm{g} / \mathrm{ml}$ anti-Creb1 (rabbit anti-Creb1 polyclonal IgG, Cat. Sc-58; Santa Cruz Biotechnology, Santa Cruz, CA, USA) or $0.25 \mu \mathrm{g} / \mathrm{ml}$ antipCreb1 (rabbit anti-pCreb1 polyclonal IgG, Cat. 06-519; Upstate Bioscientific, Lake Placid, NY, USA) overnight at $4{ }^{\circ} \mathrm{C}$. For each primary antibody, an equivalent concentration of isotype control immunoglobulin was used as a negative control. Primary antibodies were detected by secondary antibodies coupled to FITC for $1 \mathrm{~h}$ at room temperature. Optical sectioning was performed with a Bio-Rad Radiance Confocal microscope, using a Nikon Plan Apo $60 \times / 1.4$ oil emersion objective. Images were captured using Lasersharp 2000, Version 4.0 (Bio-Rad). Confocal images were equatorial optical sections. Whole section imaging was performed with mercury lamp u.v. illumination and epifluorescence on a Nikon Optiphot microscope with an Olympus DPlan Apo 40 u.v. objective. These images were subjected to deconvolution using Image-Pro plus (SharpStack; Media Cybernetics Inc., Silver Spring, MD, USA). In some experiments, embryos were counterstained with $0.1 \mu \mathrm{g}$ propidium iodide $/ \mathrm{ml}$; the FITC and propidium iodide images were merged using Image-Pro Plus.

Quantitative analysis of immunostaining in the region of the nucleus was performed using the 'Histogram' function within Image-Pro Plus. The area of the nucleus in each embryo was outlined using the 'Area of Interest' tool. The sum of staining in the area of interest was recorded.

For some images, the original gray scale images were subjected to pseudocolor transformation to provide visual representation of the staining intensity: blue (low intensity) to red (highest intensity; Image-Pro Plus). The upper and lower limitations of color division were set so that no visible color seen in negative controls.

For each experiment, embryos from each treatment were processed at the same time and in parallel. All treatments were exposed to the same preparations and dilutions of all reagents including primary and secondary antibodies. Similarly, all preparations from an experiment were examined microscopically within the same session, and used identical microscope and camera settings. All image analysis was performed in an identical manner for all embryos. All preparations were performed by the same experienced operator throughout the study. 


\section{Statistical analysis}

Statistical analysis was performed with SPSS for Windows (version 11.5; SPSS Inc., Chicago, IL, USA). Differences in the proportion of embryos developing to the blastocyst stage were analyzed by logistic regression analysis. Differences in staining intensity were assessed by ANOVA with least significance difference assessment of individual comparisons.

\section{Acknowledgment}

The authors declare that there is no conflict of interest that would prejudice the impartiality of this scientific work.

\section{References}

Bleckmann SC, Blendy JA, Rudolph D, Monaghan AP, Schmid W \& Schutz G 2002 Activating transcription factor 1 and CREB are important for cell survival during early mouse development. Molecular and Cellular Biology 22 1919-1925.

Chrivia JC, Kwok RP, Lamb N, Hagiwara M, Montminy MR \& Goodman RH 1993 Phosphorylated CREB binds specifically to the nuclear protein CBP. Nature 365 855-859.

Emerson M, Travis AR, Bathgate R, Stojanov T, Cook DI, Harding E, Lu DP \& $\mathbf{O}^{\prime}$ Neill C 2000 Characterization and functional significance of calcium transients in the 2-cell mouse embryo induced by an autocrine growth factor. Journal of Biological Chemistry 275 21905-21913.

Gonzalez G \& Montminy M 1989 Cyclic AMP stimulates somatostatin gene transcription by phosphorylation of CREB at serine 133. Cell $\mathbf{5 9}$ 675-680.

Hummler E, Cole T, Blendy J, Ganss R, Aguzzi A, Schmid W, Beermann F \& Schutz G 1994 Targeted mutation of the CREB gene: compensation within the CREB/ATF family of transcription factors. PNAS 91 $5647-5651$.

Impey S, Obrietan K, Wong S, Poser S, Yano S, Wayman G, Deloulme J, Chan G \& Storm D 1998 Cross talk between ERK and PKA is required for $\mathrm{Ca}^{2+}$ stimulation of CREB-dependent transcription and ERK nuclear translocation. Neuron 4 869-883.

Kee B, Arias J \& Montminy M 1996 Adaptor mediated recruitment of RNA polymerase II to a signal dependent activator. Journal of Biological Chemistry 271 2373-2375.

Kobayashi M \& Kawakami K 1995 ATF-CREB heterodimer is involved in constitutive expression of the housekeeping $\mathrm{Na}$,-ATPase $\alpha$ subunit gene. Nucleic Acids Research 23 2848-2855.

Korzus E, Torchia J, Rose D, Xu L, Kurokawa R, McInerney E, Mullen T, Glass C \& Rosenfeld M 1998 Transcription factor-specific requirements for coactivators and their acetyltransferase functions. Science $\mathbf{2 7 9}$ 703-707.
Latham KE \& Schultz RM 2001 Embryonic genome activation. Frontiers in Bioscience 6 D748-D759.

Leach RE, Stachecki JJ \& Armant DR 1993 Development of in vitro fertilized mouse embryos exposed to ethanol during the preimplantation period: accelerated embryogenesis at subtoxic levels. Teratology 47 57-64.

Liu Z \& Armant DR 2004 Lysophosphatidic acid regulates murine blastocyst development by transactivation of receptors for heparin-binding EGF-like growth factor. Experimental Cell Research 296 317-326.

Lu DP, Chandrakanthan V, Cahana A, Ishii S \& O'Neill C 2004 Trophic signals acting via phosphatidylinositol-3 kinase are required for normal pre-implantation mouse embryo development. Journal of Cell Science 117 1567-1576.

Mayr B \& Montminy M 2001 Transcriptional regulation by the phosphorylation-dependent factor CREB. Nature Reviews. Molecular Cell Biology 2 599-609.

O'Neill C 1997 Evidence for the requirement of autocrine growth factors for development of mouse preimplantation embryos in vitro. Biology of Reproduction 56 229-237.

Schultz RM 1989 Regulation of mouse preimplantation development: inhibition of synthesis of proteins in the two-cell embryo that require transcription by inhibitors of CAMP-dependent protein kinase. Developmental Biology 133 588-599.

Schultz RM 1993 Regulation of zygotic gene activation in the mouse. BioEssays 15 531-538.

Shaywitz AJ \& Greenberg ME 1999 CREB: a stimulus-induced transcription factor activated by a diverse array of extracellular signals. Annual Review of Biochemistry $\mathbf{6 8} 821-861$.

Stachecki JJ, Yelian FD, Leach RE \& Armant DR 1994 Mouse blastocyst outgrowth and implantation rates following exposure to ethanol or A23187 during culture in vitro. Journal of Reproduction and Fertility 101 611-617.

Stojanov T \& $\mathbf{O}^{\prime}$ Neill C 2001 In vitro fertilization causes epigenetic modifications to the onset of gene expression from the zygotic genome in mice. Biology of Reproduction 64 696-705.

Telford NA, Watson AJ \& Schultz GA 1990 Transition from maternal to embryonic control in early mammalian development: a comparison of several species. Molecular Reproduction and Development 26 90-100.

Yamamoto KK, Gonzalez GA, Biggs WH III \& Montminy MR 1988 Phosphorylation-induced binding and transcriptional efficacy of nuclear factor CREB. Nature 334 494-498.

Zhang X, Odom DT, Koo S-H, Conkright MD, Canettieri G, Best J, Chen H, Jenner R, Herbolsheimer E, Jacobsen E et al. 2005 Genome-wide analysis of CAMP-response element binding protein occupancy, phosphorylation, and target gene activation in human tissues. PNAS 102 4459-4464.

Received 31 May 2007

First decision 19 June 2007

Revised manuscript received 24 July 2007

Accepted 8 August 2007 